\title{
Betel Quid, Smoking and Alcohol Dependency among Patients with Oral Potentially Malignant Disorders and Oral Cancer in Sri Lanka; A Preliminary Case-control Study
}

\author{
Sumali R. Sumithrarachchi ${ }^{1}$, W.A.Udari.C. Pemasiri², A.M.S. Deepanie. \\ Pathiranage $^{3}$, Primali R. Jayasooriya ${ }^{4}$
}

${ }^{1,4}$ Department of Oral Pathology, Faculty of Dental Sciences, University of Peradeniya, Peradeniya, Sri Lanka. ${ }^{2,3}$ Department of Nursing, Faculty of Allied Health Sciences, University of Peradeniya, Peradeniya, Sri Lanka.

\begin{abstract}
Introduction: Oral cancer and Oral Potentially Malignant Disorders (OPMDs) arising due to risk habits like betel quid (BQ) chewing, smoking and alcohol use are preventable despite the high prevalence and poor survival rates. However, dependence to risk habits has become a barrier to prevent OPMDs and oral cancer. Objectives: This study was conducted to determine the dependence to risk habits among patients with such oral lesions, and the quitting among dependent users after diagnosis. Materials and method: A case-control study was conducted among 100 participants, 50 diagnosed with OPMDs and oral cancer from two Oral and Maxillo Facial clinics in Sri Lanka and a similar control group without any oral lesions. All participants practiced one or more risk habits such as BQ chewing, smoking and/or alcohol use. Dependence was assessed using Sinhala language translations of standardized scales such as Betel Quid Dependence Scale for BQ chewers, Fagerstrom Test for Nicotine Dependence for tobacco smokers and CAGE ("Cut down", "Annoyed", "Guilty" and "Eye opener") scale for alcohol users. Results: Dependence on habits was more among the case group (BQ-93\%; smoking-14\%; alcohol-66\%). Quitting rates of BQ and alcohol among those who were diagnosed with oral cancer [BQ $(89 \%)$, alcohol $(89 \%)$ ] were higher $(\mathrm{p}<0.05)$ than those with OPMDs [BQ $(50 \%)$, alcohol $(22 \%)$ ]. Lack of awareness due to low socio-economic status $(97 \%)$ was a significant association for BQ dependence $(p=0.01)$.Conclusion: In conclusion, development of OPMDs and oral cancer increases with dependence to BQ chewing, smoking and alcohol use. Increased awareness about OPMDs and its risk of malignancy is needed among public. Prevention of dependence to these risk habits also becomes essential.
\end{abstract}

Keywords: Alcohol dependence- Betel quid dependence- Oral cancer- Oral potentially malignant disorders

\section{Introduction}

Oral cancer, traditionally defined as squamous cell carcinoma in the lip, oral cavity and oropharynx is one of the 10 most common cancers in the world with a high annual incidence of over 300,000 diagnosed cases and an annual mortality of about 145,000 worldwide [1]. Compared to other countries, Sri Lanka has the highest mortality rate with 3 to 4 individuals dying each day due to oral cancer [2]. However, it is a preventable disease, in which betel quid (BQ) chewing, smoking and alcohol abuse are present in $90 \%$ of the cases. Oral cancer is usually preceded by Oral Potentially Malignant Disorders (OPMDs) like Oral leukoplakia (OL), Erythroplakia and Oral Submucous Fibrosis (OSF). OL is one of the most common OPMDs with approximately $90 \%$ of patients having risk habits of tobacco use [3]. Additionally, about $4 \%-36 \%$ of the cases may undergo malignant transformation [3, 4]. OSF is chronic and progressive with irreversible scarring affecting oral, oropharyngeal and esophageal mucosa due to areca-nut (AN) use $[5,6]$. $5-13 \%$ of OSF patients develop oral cancer in the back

Corresponding Author:

Prof. Primali R. Jayasooriya

Department of Oral Pathology, Faculty of Dental Sciences, University of Peradeniya, Peradeniya, 2400, Sri Lanka.

Email: primalij@yahoo.com 
ground of OSF [7].

Nicotine in tobacco is the main addictive ingredient in tobacco smoking as well as BQ with tobacco. In addition, $\mathrm{AN}$ in the BQ also contains addictive alkaloids such as arecoline. After caffeine, alcohol and nicotine, AN is the fourth most frequently consumed, psychoactive substance worldwide [8-11]. Similarly, alcoholism is a chronic, progressive and a potentially fatal disorder since discontinuation of alcohol is averted due to dependence and withdrawal symptoms [12]. In order to understand the addictive nature, a standardized quantitative measure is necessary. To assess the BQ dependency, Lee et al., [13] developed the Betel Quid Dependence Scale (BQDS) in Chinese. Herzog et al., [14] conducted a second study in English from a sample of English speaking male and female BQ chewers living in Guam. BQDS consists of three factors, namely "physical and psychological urgent need," "increasing dose" and "maladaptive use" with 16 sub items [15]. These are dichotomous questions with each "Yes" accounting for 1 point. Score 4 is considered as the cut-off point to identify dependence for BQ. The Fagerstrom Test for Nicotine Dependence (FTND) is a scale which is used to assess the nicotine dependence. FTND gives scores ranging from 0 to 10 [16]. Score 4 or more is used to identify individuals with moderate to high dependence on nicotine [17]. The 4 item CAGE ("Cut down", "Annoyed", "Guilty" and "Eye opener") questionnaire is defined as a brief, validated, effective screening device for problem drinkers [18-20]. These questions are "Yes", "No" type questions with a score ranging from $0-4$. Two or more "yes" answers are CAGE positive [20].

To the best of our knowledge, there is no published research currently available in the literature evaluating the BQ, smoking and alcohol dependency among patients with OL, OSF and oral cancer in Sri Lanka. Therefore, this study aimed to evaluate the dependency on risk habits among diagnosed patients with OL, OSF and oral cancer before and after diagnosis in comparison to dependence in those who are known to practice these habits, without any oral conditions.

\section{Materials and Methods}

A quantitative case-control study was conducted among patients with OPMDs and malignancy to determine the dependency on BQ chewing, smoking and alcohol use. The rate of dependency was compared with a control consisting of patients with risk habits but without the any oral lesions. The sample size was calculated using the open Epi software (available at http://www.openepi.com/ Menu/OE_Menu.htm) as 185 cases with OPMDs and oral cancer. However, due to time constraints and the national lockdown due to COVID-19 pandemic, a sample of 50 per each arm was selected using convenience sampling. Fifty newly, histopathologically diagnosed patients with OL, OSF and oral cancer (oral squamous cell carcinomaOSCC) having BQ chewing, smoking and/or alcohol consumption were randomly selected from the Oral and Maxillo-Facial clinics (OMF) at the Provincial General
Hospital, Kurunegala and Dental Teaching Hospital, Peradeniya. Fifty persons who practiced the above habits without the presence of any oral lesions were randomly selected from the general population as the control group.

A pre-tested, structured questionnaire was used to collect data from the participants. Part A was an interviewer administered questionnaire (administered by author no 2) consisting of socio-demographic data, habit related information and lesion details while part B was a self-administered questionnaire with BQDS, FTND and CAGE scales on dependence. Patients' clinic books and biopsy reports were used to obtain lesion related details.

Even though BQDS, FTND and CAGE are validated in English, translation and validation in Sinhala and Tamil languages was necessary. Instrument adaptation using the following steps, namely (a) forward translation, (b) expert panel back translation, (c) pre-testing (d) cognitive interviewing to get the final version was carried out. The validity and the culture adaptation of the scales were confirmed by calculating the Cronbach's $\alpha$ value; BQDS (0.959), FTND (0.70), CAGE (0.75) showing a good internal consistency.

Ethical clearance was obtained from the Ethical Review Committee of the Faculty of Allied Health Sciences (AHS/ERC/2019/084) and the Faculty of Dental Sciences (ERC/FDS/UOP/1/2020/11), University of Peradeniya.

All responses were checked for their completeness, accuracy and internal consistency analyzed using SPSS (Statistical Package for Social Science) version 21. It was done by simple descriptive methods according to the objectives of the study. Chi-square test was used to determine the associations between categorical variables. $\mathrm{p}<0.05$ was considered as significant.

\section{Results}

The case and control groups of the analyzed sample consisted of a majority (84\%) of males in each group separately, while age wise a nearly equal distribution of $44 \%$ and $56 \%$ was observed in the $\leq 60$ years and $>$ 60 years age categories of the case group. However, a majority $(80 \%)$ in the control group were $\leq 60$ years of age. When considering the socio-economic status (SES) of the sample, majority of $90 \%$ from the case group were from a low SES background. However the control group consisted of $64 \%$ and $36 \%$ of low and middle SES respectively.

Figure 1 depicts the habit related details of both groups. Accordingly 34\% (17) of participants of the case group were having all three habits of BQ chewing, smoking and alcohol abuse while only $22 \%$ (11) of the control group were engaged in all three risk habits. They were all males. When considering risk habits in the case group majority of $82 \%$ (41) were BQ chewers (Males-33, Females-8), while $58 \%$ (29) and $56 \%$ (28) had the habits of smoking and alcohol use respectively.

When comparing the risk habit dependency between the case and control groups, $93 \%$ of all BQ chewers in those who had developed oral lesions (case) have been 


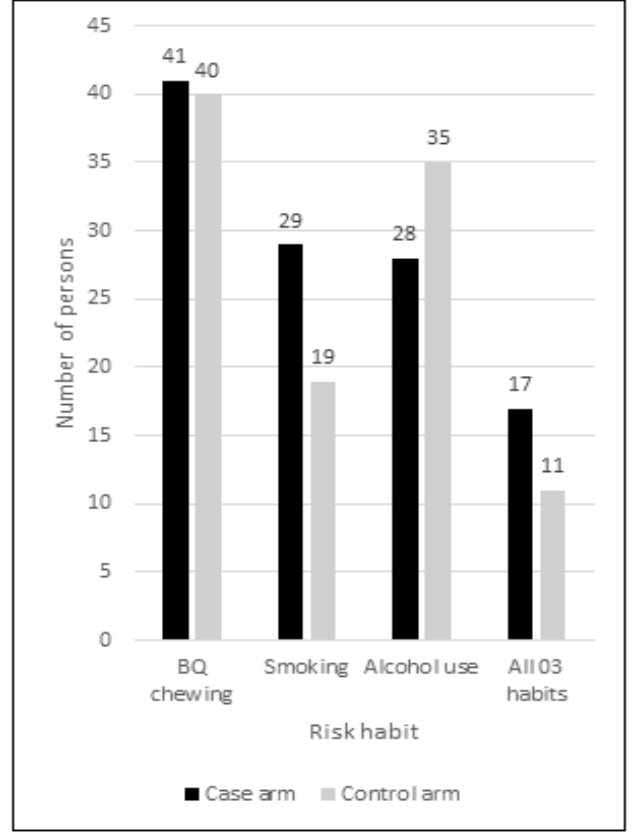

Figure 1. Risk Habits of BQ Chewing, Smoking and Alcohol Use

dependent on BQ while only $82 \%$ were dependent in the control group. Only $14 \%$ and $5 \%$ of all smokers were dependent on tobacco smoking in the case and control groups respectively. Out of the ones who had the habit of alcohol use $79 \%$ and $63 \%$ of case and control groups were dependent respectively (Table 1).

A significant finding is that among the 41 BQ chewers in the case group, majority (27) had developed oral cancer while 11 and 3 had developed OL and OSF respectively. Similarly a higher number of smokers have developed oral cancer including all dependent smokers (4) as shown in Table 1. A majority (19) of alcohol users have developed oral cancer compared to those who have developed OPMDs (6).

Table 1 shows the dependence to risk habits with different habits and combinations of habits and as shown majority of the patients with all 03 habits and BQ chewing alone had developed oral cancer in the case group.
Additionally, majority of the participants who had BQ chewing alone or in combination with smoking or alcohol use had dependence to BQ use (Table 1).

As majority of the participants had the habit of $\mathrm{BQ}$ chewing, the use of tobacco in the BQ was analyzed and it revealed that a majority $(88 \%)$ of the BQ chewers from the total sample considering both case and control groups used smokeless tobacco (SLT) as part of their quid. Accordingly, higher percentages $(90 \%)$ of subjects were dependent when SLT was added to the BQ. In the case group alone, $97 \%$ of BQ chewers with tobacco added were dependent $(\mathrm{p}=0.021)$. However, there were around $70 \%$ dependent among the persons who use BQ even without SLT use, but with areca as part of their quid.

Table 2 reveals the rate of quitting among those who were diagnosed with OL, OSF or oral cancer with regard to their initial dependence on risk habits. $76 \%$ of participants who were dependent on BQ chewing have quit after diagnosis of their conditions. Similarly out of the 4 dependent smokers, all of them have quit smoking and $64 \%$ of dependent alcohol users have quit alcohol use after diagnosis of the oral condition. However, an interesting finding is that a higher percentage of those who are dependent compared to non-dependent participants have quit their habits after diagnosis in those with BQ chewing and smoking habits.

When considering the rate of quitting with regard to the type of lesion diagnosed, $89 \%$ of participants diagnosed with oral cancer quit their habits when considering all risk habits separately. However, lesser percentages have quit habits when diagnosed with OPMDS.

Table 2 shows the association of dependence on risk habits for habit cassation in patients with OPMDs and oral cancer

Finally, when factors such as age and socio-economic status were compared with the dependency on risk habits in the case group, majority of BQ $(97 \%)$, smoking $(100 \%)$ and alcohol (82\%) dependents were from a low socio-economic background. However, majority (61\%) of BQ dependents are above 60 years of age while no such predilection was observed in smoking and alcohol dependents.

Table 1. Comparison of the Risk Habit(s) with the Dependence to Risk Habits in the Total Sample, Case Group Alone and According to the Lesion Type

\begin{tabular}{|c|c|c|c|c|c|c|c|c|c|}
\hline \multirow[t]{2}{*}{ Habit } & \multicolumn{3}{|c|}{ Total $(n=100)$} & \multicolumn{3}{|c|}{ Case group $(n=50)$} & \multicolumn{3}{|c|}{ Lesion } \\
\hline & $\begin{array}{c}\text { BQ } \\
\text { dependent }\end{array}$ & $\begin{array}{c}\text { Smoking } \\
\text { dependent }\end{array}$ & $\begin{array}{c}\text { Alcohol } \\
\text { dependent }\end{array}$ & $\begin{array}{c}\text { BQ } \\
\text { dependent }\end{array}$ & $\begin{array}{c}\text { Smoking } \\
\text { dependent }\end{array}$ & $\begin{array}{c}\text { Alcohol } \\
\text { dependent }\end{array}$ & $\mathrm{OL}$ & OSF & $\mathrm{SCC}$ \\
\hline & & & & & & & $\mathrm{n}=13$ & $\mathrm{n}=4$ & $\mathrm{n}=33$ \\
\hline BQ chewing alone & $26(29)$ & NA & NA & $15(15)$ & NA & NA & 3 & 1 & 11 \\
\hline Smoking alone & NA & $0(4)$ & NA & NA & $0(3)$ & NA & 1 & 0 & 2 \\
\hline Alcohol use alone & NA & NA & $2(3)$ & NA & NA & $1(1)$ & 0 & 0 & 1 \\
\hline BQ chewing and smoking & $4(4)$ & $0(4)$ & NA & $4(4)$ & $0(4)$ & NA & 3 & 0 & 1 \\
\hline BQ chewing and Alcohol use & $18(20)$ & NA & $14(20)$ & $5(5)$ & NA & $3(5)$ & 2 & 0 & 3 \\
\hline Smoking and Alcohol use & NA & $1(12)$ & $10(12)$ & NA & $0(5)$ & $5(5)$ & 1 & 1 & 3 \\
\hline All 03 habits & $20(28)$ & $4(24)$ & $19(28)$ & $14(17)$ & $4(13)$ & $13(17)$ & 3 & 2 & 12 \\
\hline$P$ value & 0.691 & $<0.005$ & $<0.005$ & 0.391 & $<0.005$ & $<0.005$ & & 0.94 & \\
\hline
\end{tabular}


Table 2. Association of Dependence on Risk Habits for Habit Cassation in Patients with OPMDs and Oral Cancer

\begin{tabular}{lcccccccc}
\hline Habit dependence & \multicolumn{2}{c}{ OPMDs } & \multicolumn{2}{c}{ Oral Cancer } & \multicolumn{2}{c}{ Total } & p value \\
& & $\begin{array}{c}\text { Before } \\
\text { diagnosis }\end{array}$ & $\begin{array}{c}\text { Quit habits } \\
\text { after } \\
\text { diagnosis }\end{array}$ & $\begin{array}{c}\text { Before } \\
\text { diagnosis }\end{array}$ & $\begin{array}{c}\text { Quit habits } \\
\text { after } \\
\text { diagnosis }\end{array}$ & $\begin{array}{c}\text { Before } \\
\text { diagnosis }\end{array}$ & $\begin{array}{c}\text { Quit habits } \\
\text { after } \\
\text { diagnosis }\end{array}$ \\
\hline BQ chewing & Dependent & 13 & $7(50 \%)$ & 25 & $24(89 \%)$ & 38 & $29(76 \%)$ & $p<0.05$ \\
\multirow{5}{*}{ Smoking } & Non-dependent & 1 & & 2 & & 3 & $2(67 \%)$ & \\
\multirow{5}{*}{ Alcohol use } & Dependent & 0 & $7(64 \%)$ & 4 & $16(89 \%)$ & 4 & $4(100 \%)$ & $p<0.05$ \\
& Non-dependent & 11 & & 14 & & 25 & $19(76 \%)$ & \\
& Dependent & 8 & $2(22 \%)$ & 14 & $17(89 \%)$ & 22 & $14(64 \%)$ & $\mathrm{p}<0.05$ \\
& Non-dependent & 1 & & 5 & & 6 & $5(83 \%)$ & \\
\hline
\end{tabular}

\section{Discussion}

Dependence on risk habits for OPMDs and oral cancer such as BQ chewing, smoking and alcohol use are important research areas with very few studies conducted worldwide, especially with regard to BQ dependence. As mentioned earlier, the current study was conducted to determine the dependence on these risk habits among patients who have developed oral lesions such as OL, OSF and oral cancer in comparison to a matching control group without the presence of any OPMDS or malignancy but with risk habits in order to ensure that there was no sample bias when analyzing. Hence when compared to the population statistics $[21,22]$ for prevalence of BQ chewing, smoking and alcohol use, the control group had a higher percentage. As results revealed, a higher dependence on all three risk habits were observed in the case group compared to the control group, showing that the risk of developing OPMDs and malignancies is higher, when there is a dependence on these risk habits. Lee et al., in their cross sectional study on BQ dependence and OPMDs observed that people who chewed BQ had higher prevalence rates of OPMDs, especially if they were dependent users [8]. This could be due to higher consumption patterns including quantity and frequency due to dependence and inability to control or quit the habits.

Dependence on tobacco due to presence of nicotine which is a key constituent for addiction is the main reason for smoking dependence as well as BQ dependence in which tobacco is usually added to the quid. In the current study, a majority of BQ chewers incorporated tobacco in the quid. In a multinational survey on BQ dependence, it was observed that all Nepalese chewers used tobacco-added BQ in contrast to Hunan and Hainan chewers who did not incorporate tobacco in the quid [8]. Similarly tobacco is not a popular component of the BQ among Vietnamese chewers of the north, though tobacco containing chewing habit was prevalent in the south (45.6\%) [23]. However, results of the current study revealed that about $70 \%$ of those who chewed BQ without SLT were dependent on BQ chewing as well. This is due to the presence of areca nut which constitutes addictive chemicals like arecoline. The addictive nature of arecoline is well documented in the literature [24]. However, a higher percentage (90\%) of BQ chewers with SLT were shown to be dependent using the BQDS score. This was also observed in the Nepalese BQ chewers where $99 \%$ of them were dependent users [8]. This observation is due to the addictive nature of nicotine being higher compared to arecoline and proven in the literature [25].

Even though alcohol use and addiction to it, is not a direct cause for oral malignancies, studies have indicated the synergistic effects of alcohol use along with tobacco consumption on development of oral cancer [26]. Alcohol drinking is a concomitant habitual practice with BQ chewing in several cultures and lifestyles [27]. Lee et al., found that those who drank alcohol were more likely to be dependent on BQ in Taiwan, mainland China, Malaysia, Nepal and Sri Lanka [8]. The current study reveals that there were a higher percentage of persons with all three habits in the case group compared to the control group. Additionally, majority of patients with all 3 habits in the case group had developed oral cancer further affirming the synergistic effect of alcohol use and dependence for development of oral malignancies. Ko et al., [28] conducted a hospital based study in Taiwan in which results showed that about $10 \%$ of the general population aged over 15 years were found to combine betel quid chewing with smoking and drinking at least at one time in their life. It also revealed that out of the 104 patients with oral cancer in the case group, $58(56 \%)$ of them had all three habits of betel chewing, smoking and alcohol use which is higher than the control group without lesions. This is similar to the findings of the current study where a similar trend has been revealed further affirming the combined synergistic effects of these risk habits for development of oral cancer [28].

Dependence on risk habits is also a key factor which determines the ability and determination to quit their habits. As results revealed $76 \%$ of dependent BQ chewers quit their habits after development of the lesions due to reasons such as pain and discomfort because of the oral lesion as well as due to medical interventions and advice received after diagnosis. However, the quit rates are significantly higher with the development of oral cancer than OL due to fear of death and comorbidities. A qualitative in depth study conducted among thirty oral cancer patients with BQ chewing history, interviewed the participants regarding their attempts to quit and reasons including relapses if any [10]. It revealed that BQ chewers with oral cancer typically experience four significant 
stages of behavior: pre-contemplation, contemplation, action, and maintenance. Pre-contemplation is the phase at which positive attitudes towards psychoactive effects and social effects of BQ chewing are expressed, whereas at contemplation stage the adverse effects of it become significant, which finally results in action stage of quitting. However, maintenance stage of continuing to remain without $\mathrm{BQ}$ chewing is the most difficult due to dependence and relapse. Of the 30 participants, 26 of them have quit BQ chewing and achieved the maintenance stage before the study period, while only 4 participants remained in the action stage during the study period. The participants were roughly categorized into two types: chewers who moved straight to the action stage due to oral cancer, and then possibly achieved the maintenance stage; and chewers who had tried to quit BQ but possibly experienced a cycle of chewing and quitting, then possibly achieved the maintenance stage before or after they were diagnosed with oral cancer. Hence, this study shows that despite dependence, quitting BQ chewing habits among patients diagnosed with oral cancer is relatively more prevalent due to the different stages of quitting and could be utilized to understand the findings of the current study as well. Lee et al., also showed that those who quit BQ because of oral cancer usually quit tobacco smoking and alcohol use as well, with a lesser chance of recurrence. Similarly, in our study, quitting rates of alcohol users was also significantly higher in those who were diagnosed with oral cancer. However, quitting among dependent smokers could not be analyzed accurately as there were only 4 dependent smokers in the case group and all quit their habits with the diagnosis. A controversial outcome of the current research was that comparatively a lesser percentage of non-dependent users quit habits. The small sample size was a limitation to obtain an accurate, significant association for this.

Dependence to BQ and alcohol use was more prevalent ( $97 \%$ and $82 \%$ respectively) among patients with low SES in the case group as well as control group. Similarly a corelative control study on the SES and alcohol use revealed that lower educational level, income and neighborhood SES were all associated with an increased risk for alcohol use disorders for both males and females in all ages [29]. A cross-sectional school based survey was conducted among 2200 adolescents from 26 schools of Karachi, and 874 BQ chewers were assessed for their reasons of BQ chewing and dependency [30]. The second most commonly endorsed factor amongst dependent group of BQ chewers was 'social/cultural construct' and hence validates the findings of the current study with regard to the association of low SES for BQ dependence. In one recent Sri Lankan survey, $76 \%$ of participants, primarily from lower socio-economic groups, were unaware of any ill effects from areca nut use [31].

Even though significant findings were observed in the current research, it has several limitations due to lack of considerable sample size to analyze on OSF and habit dependence as well as dependence to tobacco smoking and its association on oral lesions as well as quitting rates. As mentioned earlier, the lockdown due to the COVID-19 pandemic was one of reasons for the limited sample. Hence further research on dependence to risk habits of oral cancer using larger, randomized samples are necessary to verify these findings.

In conclusion, dependence on risk habits such as $\mathrm{BQ}$ chewing, smoking and alcohol misuse is a main factor which causes adverse health effects such as OPMDs and oral cancer. The combined synergistic effect of habits results in a higher risk of these oral conditions. Cessation of these habits also depends on the dependence, but when diagnosed with oral cancer the quitting rates are significantly higher due to fear of death and comorbidities than OPMDs. This shows the lack of awareness among public regarding OPMDs and their risk of malignancy. Hence increased awareness on adverse health effects of these risk habits need to be focused for prevention and cessation of habits and its dependence. In addition, cessation programs should target research on innovative modalities such as pharmacotherapy to overcome dependence to these habits as adjuvants to behavioral therapy.

\section{Funding}

This research did not receive any specific grant from funding agencies in the public, commercial, or not-forprofit sectors.

\section{Conflicts of Interest}

The authors declare no conflict of interest.

\section{References}

1. Rivera C. Essentials of oral cancer. Int J Clin Exp Pathol. 2015;8(9):11884-11894. Published 2015 Sep 1.

2. Jayasooriya PR, Pitakotuwage TN, Mendis BRRN, Lombardi T. Descriptive study of 896 Oral squamous cell carcinomas from the only University based Oral Pathology Diagnostic Service in Sri Lanka. BMC Oral Health. 201601 08;16(1). https://doi.org/10.1186/s12903-015-0139-y

3. Lodi G, Porter S. Management of potentially malignant disorders: evidence and critique. Journal of Oral Pathology \& Medicine. 200707 05;37(2):63-69. https://doi.org/10.1111/ j.1600-0714.2007.00575.x

4. van der Waal I. Potentially malignant disorders of the oral and oropharyngeal mucosa; terminology, classification and present concepts of management. Oral Oncology. 2009 04;45(4-5):317-323. https://doi.org/10.1016/j. oraloncology.2008.05.016

5. Jayanthi V, Probert CS, Sher KS, Mayberry JF. Oral submucosal fibrosis--a preventable disease.. Gut. 199201 01;33(1):4-6. https://doi.org/10.1136/gut.33.1.4

6. Ekanayake RP, Tilakaratne WM. Oral Submucous Fibrosis: Review on Mechanisms of Pathogenesis and Malignant Transformation. Journal of Carcinogenesis \& Mutagenesis. 2013;. https://doi.org/10.4172/2157-2518.s5-002

7. Richa, Swain N, Vibha, Gopal Ray J, Rao S. Comment on the article "Proposed clinical classification for oral submucous fibrosis" by Chandramani B. More et al.. Oral Oncology. 2012 03;48(3):290. https://doi.org/10.1016/j. oraloncology.2011.12.010

8. Lee C, Ko AM, Yen C, Chu K, Gao Y, Warnakulasuriya S, Sunarjo, Ibrahim SO, Zain RB, Patrick WK, Ko Y. Betelquid dependence and oral potentially malignant disorders 
in six Asian countries. British Journal of Psychiatry. 2012 Nov;201(5):383-391. https://doi.org/10.1192/bjp. bp.111.107961

9. Lee C, Herzog T, Chang Y. Betel Quid Dependence Scale. Neuropathology of Drug Addictions and Substance Misuse. 2016. 2016;:827-35. https://doi.org/10.1016/B978-0-12800634-4.00082-2

10. Lee C, Ko AM, Yang FM, Hung C, Warnakulasuriya S, Ibrahim SO, Zain RB, Ko Y. Association of DSM-5 Betel-Quid Use Disorder With Oral Potentially Malignant Disorder in 6 Betel-Quid Endemic Asian Populations. JAMA Psychiatry. 201803 01;75(3):261. https://doi.org/10.1001/ jamapsychiatry.2017.4307

11. Tsai M, Li T, Li C, Gritz ER, Lam C, Chiu C, Liu C, Lai C, Hsu W, Tamí-Maury I, Lu I, Lin C. Development and preliminary validation of a mandarin Chinese language questionnaire measuring betel quid dependency among adults in Taiwan. Psychiatry Research. 2019 01;271:153160. https://doi.org/10.1016/j.psychres.2018.11.027

12. Taner M, Guvenis A. Analysis of cut-off points for the CAGE Questionnaire for alcohol abuse. 2001 Conference Proceedings of the 23rd Annual International Conference of the IEEE Engineering in Medicine and Biology Society. 2001 23rd Annual International Conference of the IEEE Engineering n Medicine and Biology Society. null. https:// doi.org/10.1109/iembs.2001.1019702

13. Lee C, Chang C, Shieh T, Chang Y. Development and validation of a self-rating scale for betel quid chewers based on a male-prisoner population in Taiwan: the Betel Quid Dependence Scale. Drug Alcohol Depend. 2012;121:18-22.

14. Herzog TA, Murphy KL, Little MA, Suguitan GS, Pokhrel P, Kawamoto CT. The Betel Quid Dependence Scale: Replication and extension in a Guamanian sample. Drug and Alcohol Dependence. 2014 05;138:154-160. https://doi. org/10.1016/j.drugalcdep.2014.02.022

15. Hussain A, Zaheer S, Shafique K. Betel Quid Dependency and Associated Intrapersonal, Interpersonal, and Environmental Factors among Adolescents: A School-Based CrossSectional Survey. Substance Use \& Misuse. 201803 13;53(12):1958-1964. https://doi.org/10.1080/10826084.2 018.1447964

16. Batra A. Treatment of Tobacco Dependence. Deutsches Aerzteblatt Online. 201108 19;. https://doi.org/10.3238/ arztebl.2011.0555

17. Pérez-Ríos M, Santiago-Pérez M, Alonso B, Malvar A, Hervada X, de Leon J. Fagerstrom test for nicotine dependence vs heavy smoking index in a general population survey. BMC Public Health. 2009 Dec;9(1). https://doi. org/10.1186/1471-2458-9-493

18. Alvarez FJ, Del Rio MC. Screening for problem drinkers in a general population survey in Spain by use of the CAGE scale.. Journal of Studies on Alcohol. 1994 07;55(4):471474. https://doi.org/10.15288/jsa.1994.55.471

19. Ewing JA. Detecting alcoholism. The CAGE questionnaire. JAMA: The Journal of the American Medical Association. 1984 Oct 12;252(14):1905-1907. https://doi.org/10.1001/ jama.252.14.1905

20. Smart RG, Adlaf EM, Knoke D. Use of the CAGE scale in a population survey of drinking. Journal of Studies on Alcohol. 1991 Nov;52(6):593-596. https://doi.org/10.15288/ jsa.1991.52.593

21. Rajasuriya M. A review of interventions for smoking cessation. Sri Lanka Journal of Psychiatry. 201706 27;8(1):10. https://doi.org/10.4038/sljpsyc.v8i1.8133

22. Somatunga LC, Sinha DC, Sumenasekera P, Galapatti K, Rinchen S, Kahandaliyanage A, Mehta FR, Jayasuriya-
Dissanayake NL. Smokeless tobacco use in Sri Lanka. Indian Journal of Cancer. 2012;49(4):357. https://doi. org/10.4103/0019-509x.107729

23. Reichart PA, Nguyen XH. Betel quid chewing, oral cancer and other oral mucosal diseases in Vietnam: a review. Journal of Oral Pathology \& Medicine. 200807 08;37(9):511-514. https://doi.org/10.1111/j.1600-0714.2008.00669.x

24. Ko AM, Lee C, Ko AM, Ko Y. Betel quid dependence mechanism and potential cessation therapy. Progress in Neuro-Psychopharmacology and Biological Psychiatry. 2020 Dec;103:109982. https://doi.org/10.1016/j. pnpbp.2020.109982

25. Whiteaker P, Garcha H, Wonnacott S, Stolerman I. Locomotor activation and dopamine release produced by nicotine and isoarecolone in rats. British Journal of Pharmacology. 1995 Oct;116(3):2097-2105. https://doi. org/10.1111/j.1476-5381.1995.tb16417.x

26. Sankaranarayanan R, Duffy SW, Padmakumary G, Day NE, Krishan Nair M. Risk factors for cancer of the buccal and labial mucosa in Kerala, southern India.. Journal of Epidemiology \& Community Health. 1990 Dec 01;44(4):286-292. https://doi.org/10.1136/jech.44.4.286

27. Lee C, Ko AM, Warnakulasuriya S, Yin B, Sunarjo, Zain RB, Ibrahim SO, Liu Z, Li W, Zhang S, Kuntoro, Utomo B, Rajapakse PS, Warusavithana SA, Abdul Razak I, Abdullah N, Shrestha P, Kwan A, Shieh T, Chen M, Ko Y. Intercountry prevalences and practices of betel-quid use in south, southeast and eastern asia regions and associated oral preneoplastic disorders: An international collaborative study by asian betel-quid consortium of south and east Asia. International Journal of Cancer. 201103 08;129(7):17411751. https://doi.org/10.1002/ijc.25809

28. Ko Y, Huang Y, Lee C, Chen M, Lin L, Tsai C. Betel quid chewing, cigarette smoking and alcohol consumption related to oral cancer in Taiwan. Journal of Oral Pathology and Medicine. 1995 Nov;24(10):450-453. https://doi. org/10.1111/j.1600-0714.1995.tb01132.x

29. Calling S, Ohlsson H, Sundquist J, Sundquist K, Kendler KS. Socioeconomic status and alcohol use disorders across the lifespan: A co-relative control study. Böckerman P. PLOS ONE. 2019 Oct 17;14(10):e0224127. https://doi. org/10.1371/journal.pone.0224127

30. Hussain A, Zaheer S, Shafique K. Reasons for betel quid chewing amongst dependent and non-dependent betel quid chewing adolescents: a school-based cross-sectional survey. Substance Abuse Treatment, Prevention, and Policy. 2018 05 09;13(1). https://doi.org/10.1186/s13011-018-0154-5

31. Amarasinghe HK, Usgodaarachchi US, Johnson NW, Lalloo R, Warnakulasuriya S. Public awareness of oral cancer, of oral potentially malignant disorders and of their risk factors in some rural populations in Sri Lanka. Community Dentistry and Oral Epidemiology. 201008 23;38(6):540-548. https:// doi.org/10.1111/j.1600-0528.2010.00566.x

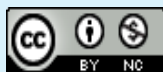

This work is licensed under a Creative Commons AttributionNon Commercial 4.0 International License. 